Article

\title{
Influence of Stress on Kinetics and Transformation Plasticity of Ferrite Transformation Based on Hysteresis Effects
}

\author{
Wenhong Ding ${ }^{1,2} \oplus$, Yazheng Liu ${ }^{3, *}$, Jianxin Xie ${ }^{1}$, Li Sun $^{4}$ and Tianwu Liu ${ }^{4}$ \\ 1 Institute for Advanced Materials and Technology, University of Science and Technology Beijing, \\ 30 Xueyuan Road, Beijing 100083, China; dingwenhong@wust.edu.cn (W.D.); jxxie@ustb.edu.cn (J.X.) \\ 2 The State Key Laboratory of Refractories and Metallurgy, Wuhan University of Science and Technology, \\ 947 Heping Avenue, Wuhan 430081, China \\ 3 School of Materials Science and Engineering, University of Science and Technology Beijing, \\ 30 Xueyuan Road, Beijing 100083, China \\ 4 HBIS Group Technology Research Institute, 385 Tiyu South Street, Shijiazhuang 050023, China; \\ sunli@hbisco.com (L.S.); liutianwu@hbisco.com (T.L.) \\ * Correspondence: lyzh@ustb.edu.cn; Tel.: +86-010-6233-3174
}

Received: 27 November 2018; Accepted: 9 January 2019; Published: 12 January 2019

\begin{abstract}
Transformation plasticity and kinetics play an essential role in the prediction of residual stresses resulting from transformation. This paper is devoted to the investigation of the influence of stress on the kinetics and transformation plasticity of ferrite for H420LA steel. It has been shown that under small external stresses, lower than the yield stress of the weaker phase, the ferrite transformation is inhibited at the beginning of the transformation in the continuous cooling process and the mechanical stabilization of austenite is observed, due to transformation hysteresis effects. This phenomenon affects the metallurgical and mechanical behaviors of the transformation progress. However, most existing models ignore these effects, leading to deviations in the description of transformation plasticity during the transformation progress. Considering the hysteresis effects, the micromechanical model for kinetics and transformation plasticity is reexamined. A general formulation of austenite decomposition kinetics accounting for these effects is developed to better describe the phase transformation under a continuous cooling process. In addition, the influence of hysteresis effects on the evolution of transformation plasticity is analyzed. Consideration of the hysteresis effects decreases the discrepancy between the calculated and experimental values. This will allow better prediction of residual stresses in the thermomechanically controlled processes.
\end{abstract}

Keywords: transformation kinetics; transformation plasticity; thermomechanical processes; continuous cooling

\section{Introduction}

There are two types of interactions between stress and phase transformation: metallurgical and mechanical [1]. Metallurgical interactions affect the kinetics and modify the morphologies of the phase transformation products. Mechanical interactions are related to transformation plasticity, which is a kind of plastic strain that occurs under uniaxial stress during metallurgical transformations [2]. These two interactions dominate the residual stress distribution inside the material during continuous cooling. Therefore, an accurate description of the kinetics and transformation plasticity is crucial for understanding the microscopic mechanism of residual stress.

The effects of stress on the kinetics and transformation plasticity continue to receive significant research attention. The work of Johnson and Mehl [3] and Avrami [4,5] led to significant advances in 
the prediction of the kinetics and isothermal transformations. They studied the relationship between the percentage of the new phase formed and time and proposed general relations for the kinetics. Following this work, Denis et al. [1] and Fernandes et al. [6] introduced the influence of external stress and revised the Johnson-Avrami-Mehl equation. For the continuous cooling process, the Scheil equation [1] has been widely used to calculate the early stages of the transformation from the incubation periods during isothermal transformations. However, it is difficult to predict the volume fraction of the transformation product accurately with this equation. Therefore, a kinetics function for the prediction of austenite decomposition during the continuous cooling of steels must be developed.

From a microstructural viewpoint, two mechanisms are usually considered to explain transformation plasticity: the Magee mechanism (1966) [7] and Greenwood-Johnson mechanism $(1965)[8,9]$. For diffusional metallurgical transformations, the Greenwood-Johnson mechanism seems to be dominant over the Magee mechanism [10]. Greenwood and Johnson first established the relationship between the external stress and phase transformation plasticity. Following their work, a more complete formulation that accounted for the transformation plasticity progress and introduced transformation plasticity kinetics was proposed by Abrassart, Desalos, and Leblond et al. [11,12] However, it was assumed that the external stress does not affect the transformation kinetics in these studies, and the transformation hysteresis effects were ignored.

In recent years, with the growing problem of residual stress in the welding and quenching process, the research on transformation plasticity and kinetics has gradually shifted from theoretical research to application [13-15]. Transformation plasticity has been already formulated in a system of constitutive equations, integrated in the commercial software for predicting the residual stress distribution $[16,17]$. However, since the microalloyed high strength steels are usually composed of multi-phase microstructure, the study of multi-phase microstructure transformation plasticity has become a new focus $[18,19]$.

The purpose of the present work was to investigate the influence of transformation hysteresis effects on the kinetics and transformation plasticity of the ferrite transformation for H420LA steel in EN-10268. Moreover, a new kinetics model that provides a better description of the experimental results under continues cooling was developed. It will then be shown that considering hysteresis effects may remove the singularity of the transformation plasticity at the beginning of the ferrite transformation.

\section{Materials and Methods}

The experiments presented in this paper were carried out using H420LA hot rolled steel, provided by the HBIS Group Technology Research Institute (Shijiazhuang, China), which had the chemical composition shown in Table 1. Thermomechanical simulations experiments were performed on a Gleeble 3500 simulator (Dynamic Systems Inc. Poestenkill, NY, USA). The specimens were cylindrical with diameters of $6 \mathrm{~mm}$ and lengths of $15 \mathrm{~mm}$ (Figure 1).

Table 1. Chemical composition of H420LA steel (wt \%).

\begin{tabular}{cccccccc}
\hline $\mathbf{C}$ & $\mathbf{M n}$ & $\mathbf{S i}$ & $\mathbf{N b}$ & $\mathbf{C u}$ & $\mathbf{C r}$ & $\mathbf{T i}$ & $\mathbf{F e}$ \\
\hline 0.068 & 0.7 & 0.05 & 0.026 & 0.015 & 0.037 & 0.02 & Bal. \\
\hline
\end{tabular}

Two tests were performed: a free dilatometry test and a loading dilatometry test, and the thermal cycle process was the same in both experiments, as shown in Figure 2. The specimens were heated up $960{ }^{\circ} \mathrm{C}$ with the heating rate of $10^{\circ} \mathrm{C} / \mathrm{s}$, held at a $960^{\circ} \mathrm{C}$ for $5 \mathrm{~min}$ (austenitization), and then cooled to $25^{\circ} \mathrm{C}$. To achieve desired metallurgical phase, the cooling rate was $-10{ }^{\circ} \mathrm{C} / \mathrm{s}$ for all tests. In the free dilatometry process, specimens underwent temperature cycling only. In the loading dilatometry process, a stress was applied to the specimens at about $810^{\circ} \mathrm{C}$, which is before the onset temperature $\left(800^{\circ} \mathrm{C}\right)$ of the ferrite formation during cooling. The stress was maintained for the whole process. Several loading dilatometry tests were performed with tensile and compression uniaxial stresses. The 
magnitudes of the applied stresses were 15, 30, and $45 \mathrm{MPa}$, which are less than $69 \mathrm{MPa}$, the yield stress of the austenitic phase of H420LA at $800{ }^{\circ} \mathrm{C}$, basing on tensile test results.

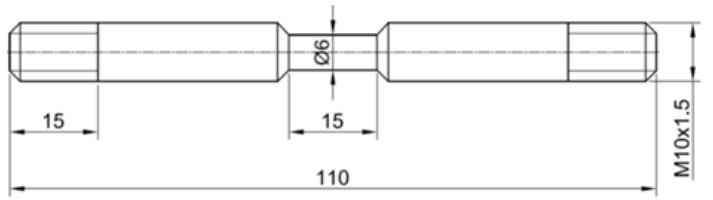

(a)

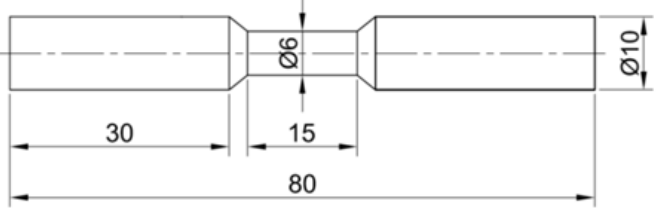

(b)

Figure 1. Specimen for experiment, (a) tensile specimen, (b) compression specimen (unit: mm).

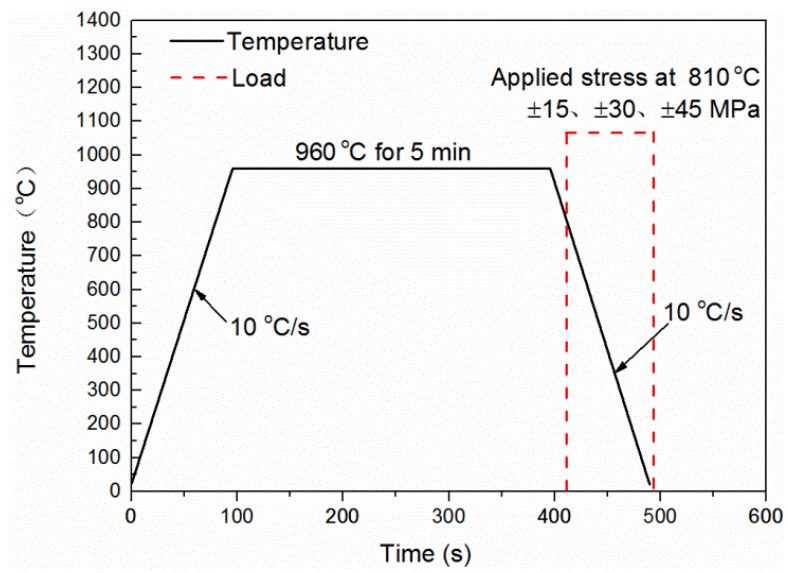

Figure 2. Heating cycle and loading procedure during tests.

\section{Results}

\subsection{Initiation Temperature}

The effects of stress on the ferrite transformation were experimentally investigated. The experimental results are shown in Figure 3 (tensile tests in Figure 3a and compression tests in Figure 3b).
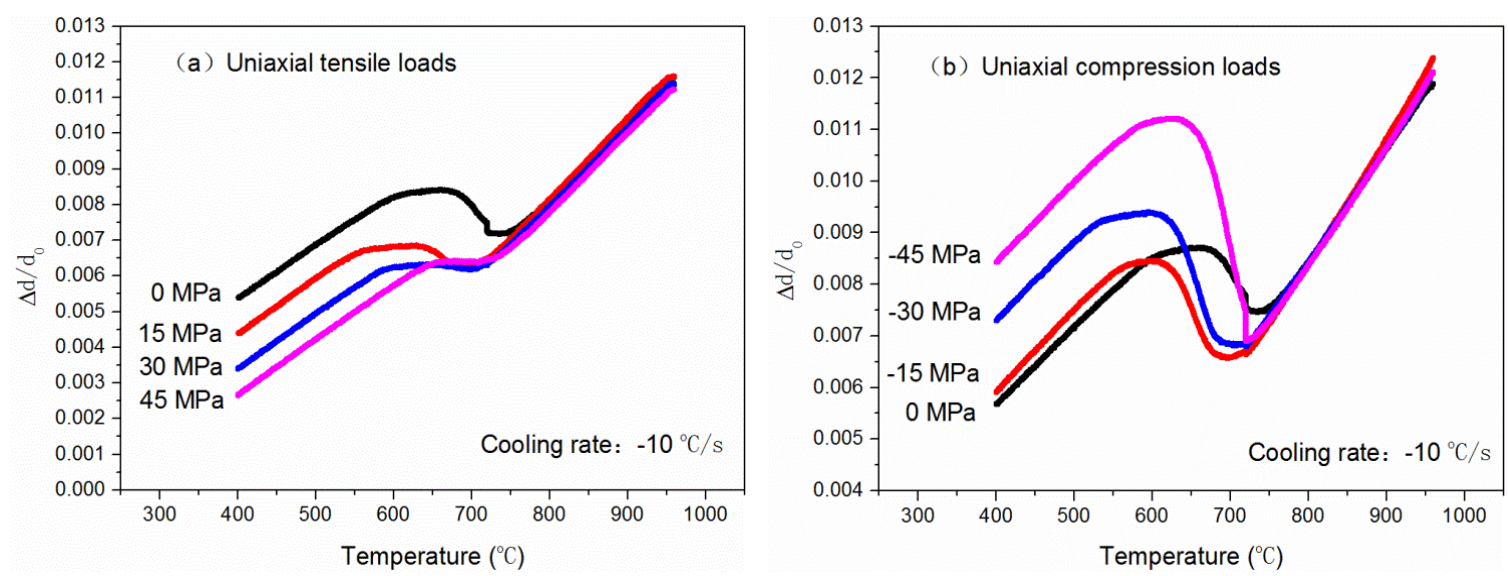

Figure 3. Total strain vs. temperature under different uniaxial tensile/compressive loads. 
At the beginning of transformation, the ferrite formation was inhibited by the uniaxial load in the elastic regime. Compressive and tensile stresses reduce the initial temperature of ferrite transformation. Herein, this phenomenon is referred to as the transformation hysteresis effect.

The onset temperature and hysteresis time of the ferrite transformation under different uniaxial loads are shown in Table 2. These results indicate that the transformation hysteresis effects under small external stresses were more significant, and as the load increased, the hysteresis time was gradually reduced. Under a $15 \mathrm{MPa}$ stress, the hysteresis time of the phase change was $7.5 \mathrm{~s}$. When the load was increased to $45 \mathrm{MPa}$, the hysteresis time was shortened to $2.3 \mathrm{~s}$. The effect of compressive stress was similar to that of tensile stress.

Table 2. Onset temperature and hysteresis time of ferrite formation under different uniaxial loads.

\begin{tabular}{cccccccc}
\hline $\mathrm{F}(\mathrm{MPa})$ & 0 & -15 & -30 & -45 & 15 & 30 & 45 \\
Onset Temperature $\left({ }^{\circ} \mathrm{C}\right)$ & 802 & 742 & 749 & 780 & 727 & 756 & 779 \\
Hysteresis temperature $\left({ }^{\circ} \mathrm{C}\right)$ & 0 & 60 & 53 & 22 & 75 & 46 & 23 \\
Hysteresis time $(\mathrm{s})$ & 0 & 6 & 5.3 & 2.2 & 7.5 & 4.6 & 2.3 \\
\hline
\end{tabular}

\subsection{Accelerated Phase Transition}

According to the lever rule [20], the volume fraction of the $\alpha$ phase (ferrite phase) $\xi$ can be calculated experimentally according to the following equation:

$$
\xi=\frac{\left(\frac{\Delta d}{d_{0}}\right)^{\mathrm{T}}-\alpha_{A}\left(T_{i}-T^{\prime}\right)}{\beta_{F}^{T^{\prime}}+\left(\alpha_{F}-\alpha_{A}\right)\left(T_{i}-T^{\prime}\right)}
$$

where $\left(\frac{\Delta d}{d_{0}}\right)^{\mathrm{T}}$ is the (total) radial expansion ratio measured experimentally during the loading dilatometry test, $T^{\prime}$ is the reference temperature at which austenite is completely transformed into ferrite/pearlite (about $422{ }^{\circ} \mathrm{C}$ in this test), $T_{i}$ is the temperature at any point during the phase change process, and $\alpha_{F}$ and $\alpha_{A}$ are the dilation coefficients depending on the temperature of the $\alpha$ and $\gamma$ phases (austenite phase), respectively. These parameters can be obtained from the free dilatometry experiments.

The volume fractions of ferrite transformation under continuous cooling were calculated from the experimental results, and some of them are presented in Figure 4 (tensile tests in Figure 4a and compression tests in Figure 4b). These results show that the kinetics of ferrite phase transformation were accelerated by both uniaxial tensile and compressive loading. Moreover, the acceleration effect under large stresses was more significant than that under small stresses. Similar conclusions were observed from other steels by Denis et al. [1]. In this work, under free stress conditions, it took about $25.7 \mathrm{~s}$ to complete a $95 \%$ ferrite transformation. When the load was increased to $45 \mathrm{MPa}$ the transformation time was shortened to $16.3 \mathrm{~s}$. The relationship between the transformation time and uniaxial loads is presented in Table 3. It is obvious that the transformation time decreased as the applied stress increased and the applied stress shortened the phase change time.

Table 3. The relationship between transformation time and uniaxial loads.

\begin{tabular}{cccccccc}
\hline$\sigma(\mathrm{MPa})$ & 45 & 30 & 15 & 0 & -15 & -30 & -45 \\
\hline$\Delta t(\mathrm{~s})$ & 16.3 & 18.9 & 22.3 & 25.7 & 22 & 21 & 19.2 \\
\hline
\end{tabular}

The austenite decomposition is an interface-controlled diffusion transformation. Thus, the main factor affecting the transformation velocity is the orientation relationship between the grain boundaries. Ferry and Humphreys [21] studied the mobility for the low angle boundaries of Al- $0.05 \%$ $\mathrm{Si}$, which showed that the mobility was increased 13 times by increasing misorientation by only $2-5^{\circ}$. Gottstein et al. [22] reached the same conclusion using an activated enthalpy viewpoint, concluding 
that the activation enthalpy of the high-angle grain boundary was significantly smaller than that of the low-angle grain boundary, and the migration activity at the phase interface was greater. The stress increases the grain boundary angle as well as the moving speed of the interface, and the transformation is accelerated.
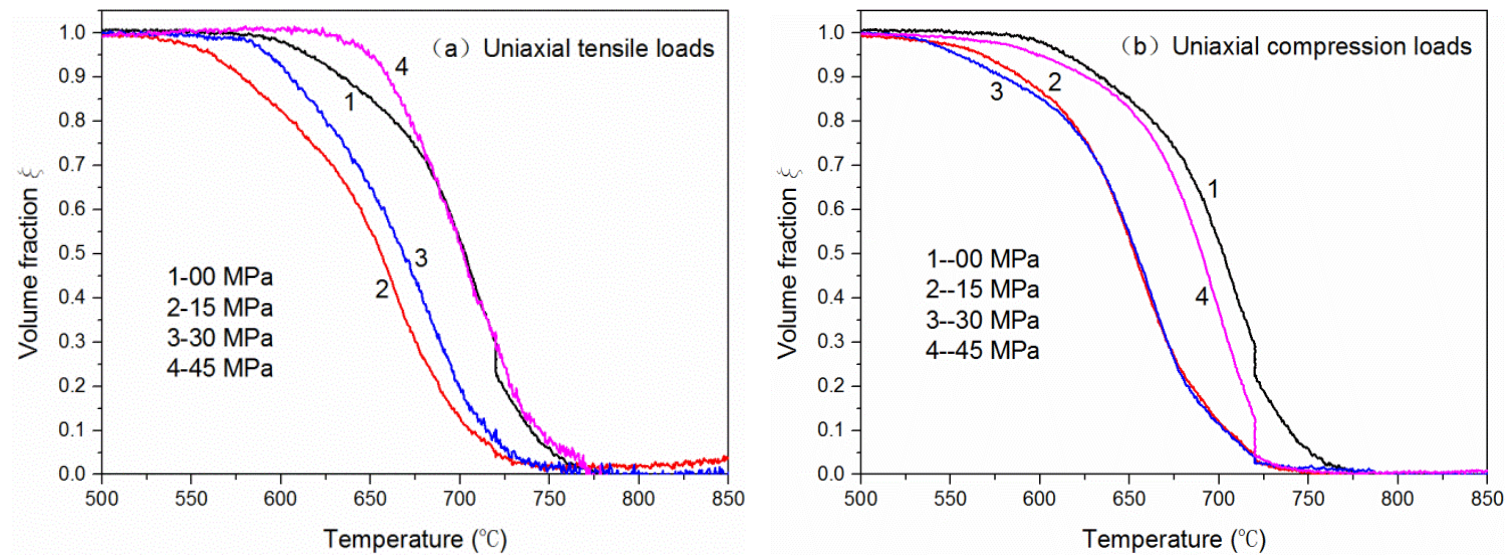

Figure 4. Effect of different uniaxial tensile/compressive stress on transformation kinetics.

\subsection{Transformation Plasticity Coefficient}

To experimentally investigate the relationship between uniaxial loads and transformation plasticity, we considered the deformation measured in the free dilatometry test to be purely thermo-metallurgical. The total observed macroscopic deformation in the applied stresses dilatometry test is the sum of four contributions: thermo-metallurgical, elastic, classical plasticity, and transformation plasticity [10], expressed as follows:

$$
\left(\frac{\Delta d}{d_{0}}\right)^{\mathrm{T}}=\left(\frac{\Delta d}{d_{0}}\right)^{\mathrm{thm}}+\left(\frac{\Delta d}{d_{0}}\right)^{\mathrm{e}}+\left(\frac{\Delta d}{d_{0}}\right)^{\mathrm{p}}+\left(\frac{\Delta d}{d_{0}}\right)^{\mathrm{tp}}
$$

where $\left(\frac{\Delta d}{d_{0}}\right)^{\mathrm{T}}$ is the total macroscopic deformation, $\left(\frac{\Delta d}{d_{0}}\right)^{\mathrm{e}}$ is the elastic component, $\left(\frac{\Delta d}{d_{0}}\right)^{\mathrm{p}}$ is the classical plasticity component, $\left(\frac{\Delta d}{d_{0}}\right)^{\text {thm }}$ is the thermo-metallurgical component, and $\left(\frac{\Delta d}{d_{0}}\right)^{\text {tp }}$ is the transformation plasticity component.

The thermo-metallurgical component can be measured experimentally from the free dilatometry tests. The elastic component can be calculated using Hooke's law:

$$
\left(\frac{\Delta d}{d_{0}}\right)^{\mathrm{e}}=-\mu \varepsilon=-\mu\left(\frac{\sigma}{E^{T}}\right)
$$

where $\sigma$ is uniaxial stress, $\mu$ is coefficient, here $\mu=0.3$, and $E^{T}$ is the Young's modulus. Assuming a linear mixture rule, the Young's modulus of the phase mixture $(\alpha+\gamma)$ has the following form:

$$
E^{T}=E_{F}^{T} \xi+E_{A}^{T}(1-\xi)
$$

where $E_{F}^{T}$ and $E_{A}^{T}$ are the Young's moduli of the $\alpha$ and $\gamma$ phases, respectively.

In the elastic regime, specimens do not exhibit classical plastic deformation. Thus, Equation (2) can be rewritten as the following equation:

$$
\left(\frac{\Delta d}{d_{0}}\right)^{\mathrm{tp}}=\left(\frac{\Delta d}{d_{0}}\right)^{\mathrm{T}}-\left(\frac{\Delta d}{d_{0}}\right)^{\mathrm{N}}-\mu\left(\frac{\sigma}{E_{F}^{T} \xi+E_{A}^{T}(1-\xi)}\right)
$$


Transformation plastic strain in loading direction will be given by:

$$
\varepsilon^{\mathrm{tp}}=-2\left(\frac{\Delta d}{d_{0}}\right)^{\mathrm{tp}}=-2\left[\left(\frac{\Delta d}{d_{0}}\right)^{\mathrm{T}}-\left(\frac{\Delta d}{d_{0}}\right)^{\mathrm{N}}-\mu\left(\frac{\sigma}{E_{F}^{T} \xi+E_{A}^{T}(1-\xi)}\right)\right]
$$

The transformation plastic strain can be obtained from the data using Equation (6), as shown in Figure 5. Qualitatively, it can be observed tensile stresses lead to positive transformation plastic strains and vice versa. In the low stress range $( \pm 15 \mathrm{MPa})$, the tensile stress had a more significant effect on the transformation plasticity, which was three times greater than that of the compressive stress. With the increase of the stress magnitude, the transformation plasticity of the compressive stress gradually exceeded that of the tensile stress. Under a $-45 \mathrm{MPa}$ stress, the transformation plasticity of compressive stress was $19 \%$ higher than that of the tensile stress.

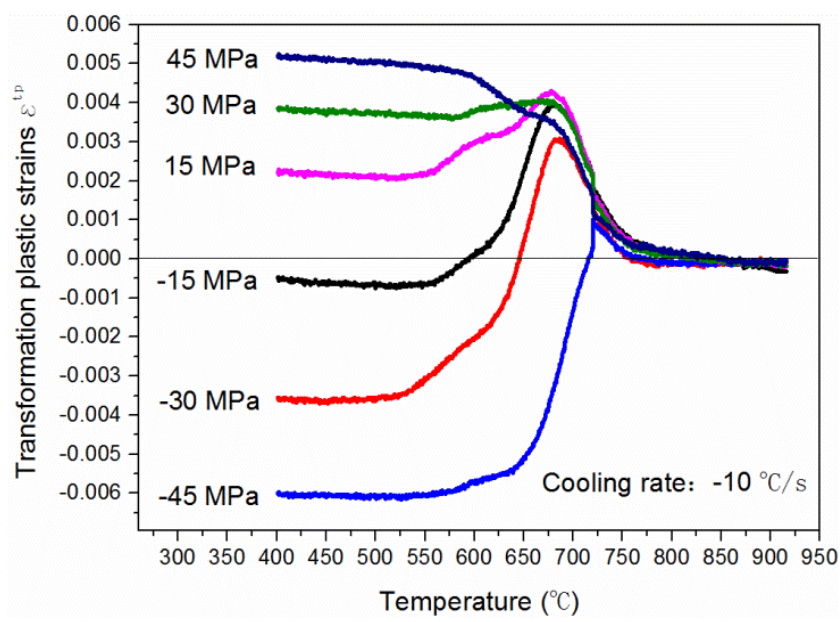

Figure 5. Curves of transformation plastic strains vs. temperature.

Based on the Greenwood-Johnson mechanism, transformation plastic strains often exhibit the following general form $[11,23]$ :

$$
\varepsilon^{\mathrm{tp}}=\mathrm{k} \sigma f(\xi)
$$

where $\mathrm{k}$ is the transformation plasticity coefficient, $\sigma$ is the uniaxial stress, $\xi$ is the volume fraction proportion of the new phase, $f(\xi)$ is a function that expresses the kinetics of the plastic transformation. Transformation plasticity is a special type of plasticity that occurs under uniaxial loads during transformation. Therefore, when $\xi=0$, transformation plasticity is equal to zero, and when the transformation ends, it is equal to one, described by the following equation:

$$
f(\xi)= \begin{cases}0 & \text { if } \xi=0 \\ 1 & \text { if } \xi=1\end{cases}
$$

If the applied load is less than the yield stress below the temperature at which the phase transition begins, and the applied load does not produce any classical plastic deformation, the phase transformation plasticity coefficient $\mathrm{k}$ can be considered to be constant, calculated by the following equation:

$$
\mathrm{k}=\frac{\varepsilon^{\mathrm{tp}}}{\sigma}
$$

Under these conditions, several tensile and compressive tests, in which the applied stress magnitudes were equal to $0,15,30$, and $45 \mathrm{MPa}$, were performed. The average value of transformation plasticity coefficient $\mathrm{k}$ obtained from the experimental results was $1.219 \times 10^{-4} \mathrm{MPa}^{-1}$ (see Table 4 and Figure 6). 
Table 4. Experimental identification of the transformation plasticity parameter.

\begin{tabular}{cccccccc}
\hline$\sigma(\mathrm{MPa})$ & 0 & -15 & -30 & -45 & 15 & 30 & 45 \\
$\mathcal{E}^{\mathrm{tp}}(\%)$ & 0 & -0.145 & -0.362 & -0.611 & 0.209 & 0.374 & 0.512 \\
$k\left(10^{-4} \mathrm{MPa}^{-1}\right)$ & 0 & 0.964 & 1.21 & 1.36 & 1.39 & 1.25 & 1.14 \\
\hline
\end{tabular}

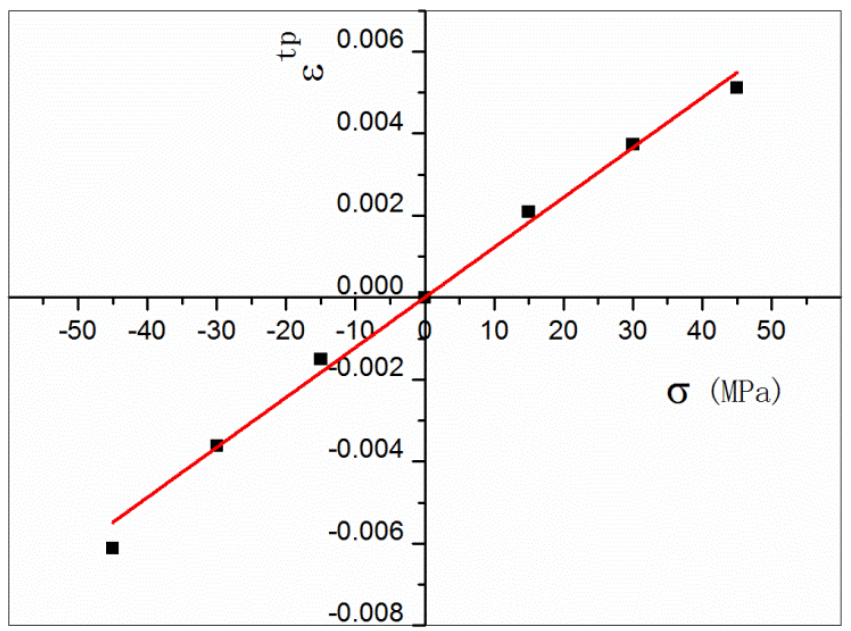

Figure 6. Relationship between transformation plastic strains and uniaxial loads.

\section{Discussion}

\subsection{Modeling the Transformation Hysteresis Effects}

From the applied stress dilatometry tests, transformation hysteresis effects were apparent under small external stresses, and the hysteresis time gradually shortened as the stress increased, as shown in Figure 7. The tests showed that the hysteresis times of transformation are similar when tensile and compressive stresses with same magnitude are loaded. From a modeling viewpoint, the transformation hysteresis effects function caused by the equivalent stress can be fitted according to the experimental data using Equation (10):

$$
F_{S}=719+1.2667 \bar{\sigma}
$$

where $F_{S}$ is onset temperature of ferrite transformation, $\bar{\sigma}$ is the equivalent stress.

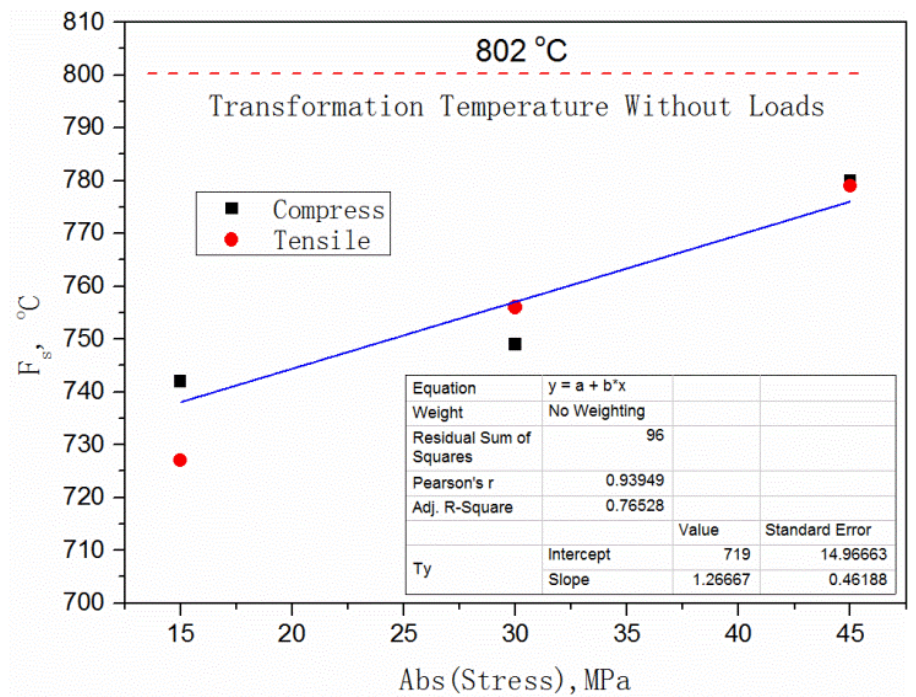

Figure 7. The effect of applied stress on initial temperature of ferrite transformation, Fs. 
$\mathrm{Xu}[24]$ investigated the martensitic transformation under external stress and found that the austenite was mechanically stabilized due to the pre-deformation of austenite, which can lower the Martensitic transformation onset temperature, Ms (Martensite start temperature), and inhibit the martensitic transformation. $\mathrm{Xu}$ [25] proposed that this phenomenon may result from the hindrance of directional growth of martensite by dislocations formed during deformation.

The results presented herein also show mechanical stabilization of austenite in the early stage of ferrite transformation under an applied stress in the elastic regime, but the mechanism may be different from the martensitic transformation. In addition, more tests are necessary to determine whether this phenomenon persists when the applied load is greater than the yield strength of the parent phase.

\subsection{Analysis of Transformation Hysteresis Effects}

The onset temperature of transformation is determined by two key factors, namely the critical nucleation and activation energies. When the new phase nucleates homogeneously in the parent phase, its critical nucleation $\Delta G^{*}$ is determined by the transformation free energy, elastic strain energy, and interface energy [26], expressed as follows:

$$
\Delta G^{*}=\frac{16 \pi \rho^{3}}{3\left(\Delta G_{V}+\Delta G_{E V}\right)^{2}}
$$

where $\Delta G^{*}$ is the critical nucleation energy, $\Delta G_{V}$ is the phase change free energy per unit volume, $\Delta G_{E V}$ is the elastic strain energy per unit volume, and $\rho$ is the unit interface energy between the new and parent phases.

The homogeneous nucleation rate, $I$, can be calculated by the following equation [26]:

$$
I=K d^{* 2} \exp \left(-\frac{\Delta G^{*}+Q}{m T}\right)
$$

where $\mathrm{K}$ is a constant independent of temperature, $d^{*}$ is the size of the critical core, $Q$ is the diffusion activation energy, $\mathrm{m}$ is the Boltzmann constant, and $T$ is the phase change temperature.

Under small external stresses, a mismatch between the austenite grains can be accommodated by elastic coherence. At this time, the unit interface energy $\rho$ remains unchanged, and the elastic strain energy $\Delta G_{E V}$ rises, resulting in a decrease in $\left|\Delta G_{V}+\Delta G_{E V}\right|$ and an increase in the critical nucleation energy $\Delta G^{*}$. According to Equation (12), the nucleation rate, I, will decrease under this condition. Therefore, applying a small external stress on the specimen during the transformation process leads to the occurrence of transformation hysteresis effects.

As the applied stress is gradually increased, the lattice spacing of the parent phase will continuously expand, resulting in a decrease in the activation energy $Q$, which controls the atom migration from the parent phase to the new phase. Therefore, in the elastic regime, the mechanical stabilization of austenite under small stresses is more obvious. Moreover, as the load increases, the transformation hysteresis effects are gradually weakened.

\subsection{Kinetics Function Based on Hysteresis Effects}

In typical treatments, the Johnson-Avrami-Mehl equation [3,4] is a general function for describing the isothermal transformation. However, non-isothermal transformations are more common in industrial applications. Therefore, it is necessary to develop a function similar to the Johnson-Avrami-Mehl equation to describe the kinetics during continuous cooling process. For this reason, considering the influence of temperature and applied stress on the volume fraction of the new phase, the following empirical kinetics function for ferrite transformation under continuous cooling conditions is proposed:

$$
\xi=1-\exp \left[-\mathrm{b}\left(F_{S}-\mathrm{T}\right)^{n}\right]
$$

where $\mathrm{n}$ and $\mathrm{b}$ are kinetics constants. The kinetics constant $\mathrm{n}$ is mainly influenced by the applied stress. 
Considering the hysteresis effects, substituting Equation (10) into Equation (13), $\xi$ is given by the following equation:

$$
\xi=1-\exp \left[-\mathrm{b}(719+1.2667 \bar{\sigma}-\mathrm{T})^{n}\right]
$$

The volume fraction of ferrite transformation was fitted using Equation (14) to obtain $\mathrm{n}$ and b values under different loads. The fitting results for 15, 30, and $45 \mathrm{MPa}$ loads are presented in Figure 8a-c and Equations (15)-(17), respectively. This new kinetics equation for ferrite transformation agrees well with the experimental values. Using this model, the volume proportion of the product phase under external stresses in the continuous cooling process can be described accurately.

$$
\begin{aligned}
& \xi=1-\exp \left[-1.09 * 10^{-4}(738-\mathrm{T})^{1.97}\right] \\
& \xi=1-\exp \left[-2.78 * 10^{-5}(757-\mathrm{T})^{2.27}\right] \\
& \xi=1-\exp \left[-1.59 * 10^{-5}(776-\mathrm{T})^{2.49}\right]
\end{aligned}
$$
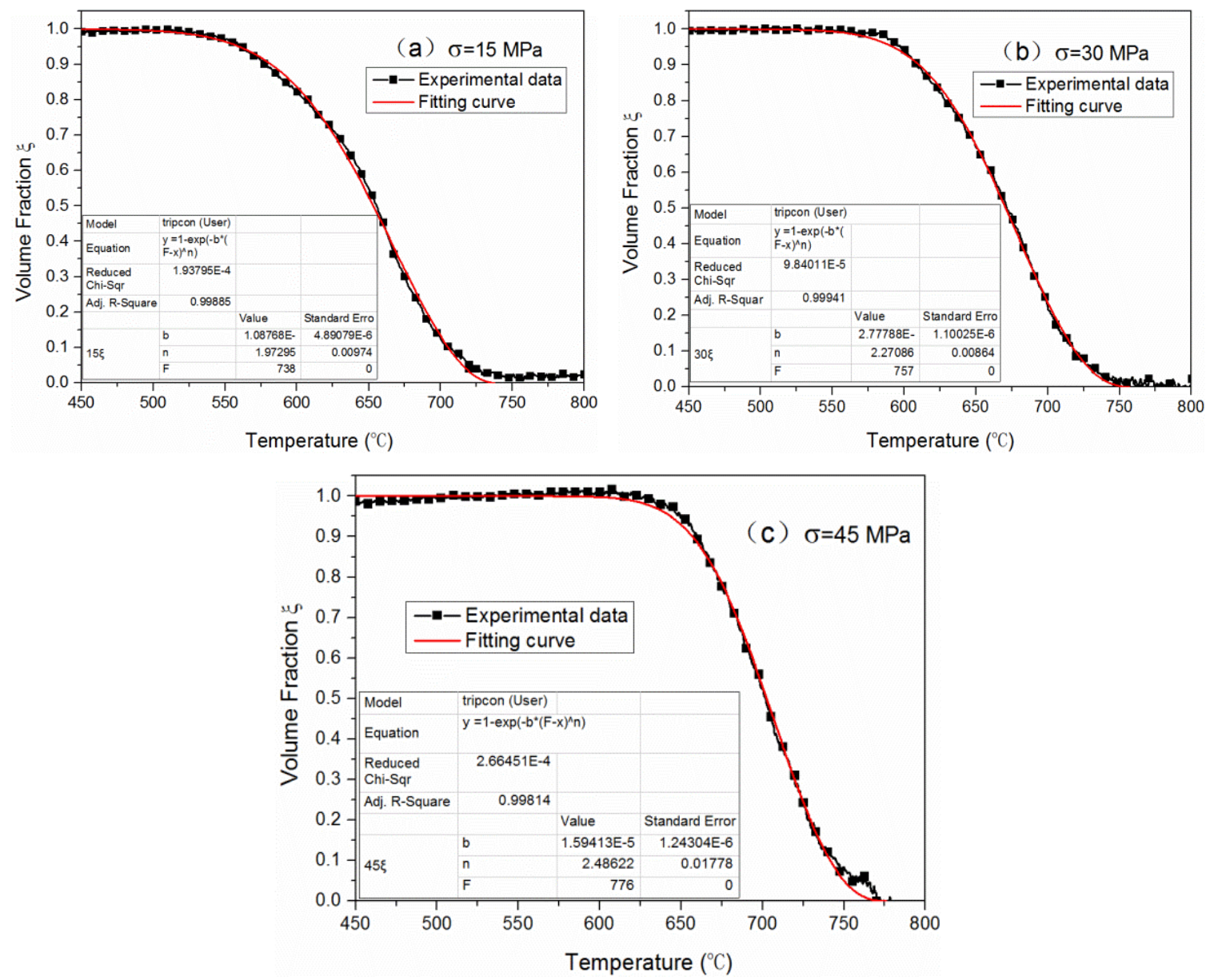

Figure 8. Fitting results of ferrite transformation kinetics for H420LA steel.

\subsection{Hysteresis effects on Transformation Plasticity}

To estimate the transformation plasticity evolution, transformation plasticity kinetics must be considered, according to Equation (7). Table 5 shows the transformation plasticity kinetics functions $f(\xi)$ proposed by Abrassart, Desalos, and Leblond et al. [11,12]. 
Table 5. Transformation plasticity kinetics functions.

\begin{tabular}{cccc}
\hline - & Leblond & Abrassart & Desalos \\
\hline$f(\xi)$ & $\xi(1-\operatorname{In} \xi)$ & $3 \xi-2 \xi^{1.5}$ & $\xi(2-\xi)$ \\
\hline
\end{tabular}

The transformation plasticity under small stresses was calculated based on the empirical transformation plasticity kinetics model and compared with the obtained experimental result. Some results are presented in Figure 9. Figure 9a,b show the transformation plasticity curves under a uniaxial tensile stress $45 \mathrm{MPa}$ and compressive stress $-45 \mathrm{MPa}$, respectively.
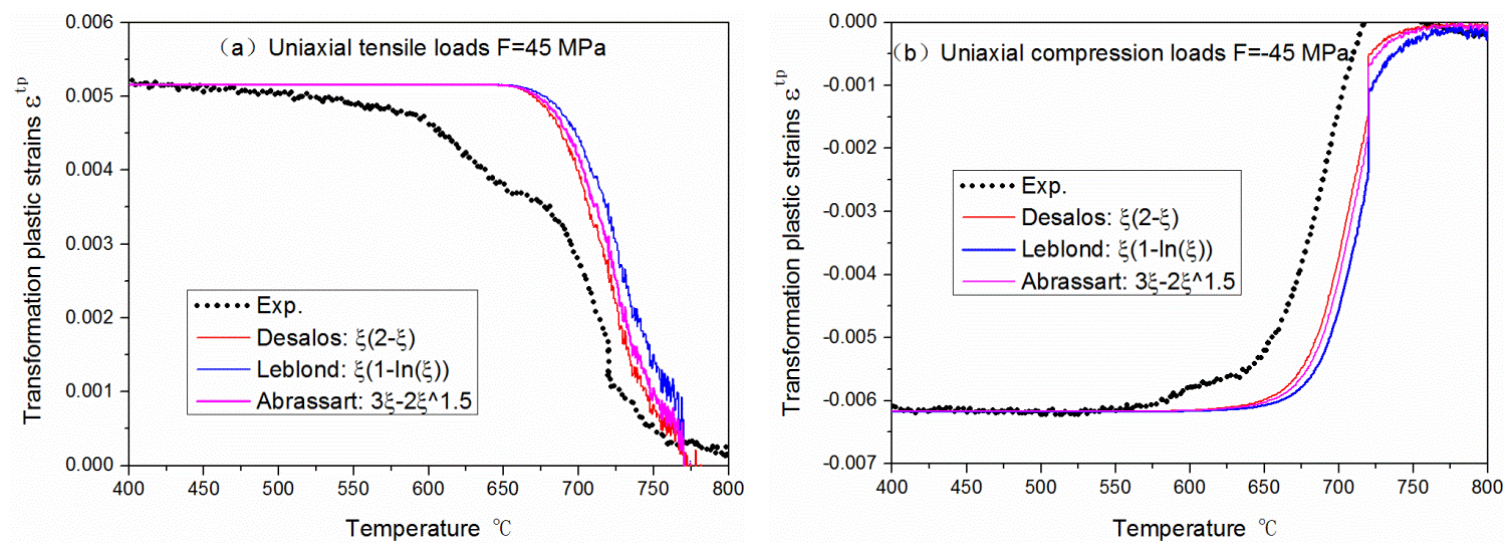

Figure 9. The comparison of calculated and experimental values for ferrite transformation in H420LA steel.

Compared to Abrassart's and Leblond's empirical model, Desalos's function best represents the experimental data, whereas Abrassart's and Leblond's function largely overestimates the experimental results. However, one significant discrepancy is apparent for Desalos's function: the phase transformation plasticity at the initial stage of transformation is overestimated. At the end of the phase change, the transformation plasticity magnitude calculated by the three empirical models are all consistent with the experimental results.

The differences between the experimental and calculated values, can be attributed to the transformation plasticity kinetics. These classical models, such as the widely used Desalos's model, assumed that uniaxial stress does not influence the transformation plasticity kinetics. The experimental results in this work (Figure $3 a, b$ ) showed that the transformation characteristics could be modified under small applied stresses (elastic regime). This becomes more significant under small applied stresses, such as $\pm 15 \mathrm{MPa}$. In addition, applied stresses also modify the starting and ending temperatures of ferrite transformation, as shown in Figure 4a,b. Indeed, applied stresses influence the transformation plasticity kinetics. Consequently, using the free dilatometry data to calculate the transformation plasticity, as used in Desalos's model, leads to overestimation of the transformation plasticity at the beginning of the transformation.

To eliminate the effect of hysteresis on the evolution of transformation plasticity, the initial transformation temperature of Desalos's empirical model [11] is reduced by $23{ }^{\circ} \mathrm{C}$ (for $45 \mathrm{MPa}$ uniaxial tensile load) and $22{ }^{\circ} \mathrm{C}$ (for $-45 \mathrm{MPa}$ uniaxial compression load), according to the experimental results shown in Table 2. The calculated value of the compressive stress agrees with the experimental value (Figure 10b), and the difference between the calculated and experimental values for the tensile stress is also reduced (Figure 10a). Therefore, accounting for the influence of the transformation hysteresis effects enables a better description of ferrite transformation under small external stresses and removes the deviations at the beginning of the transformation. 

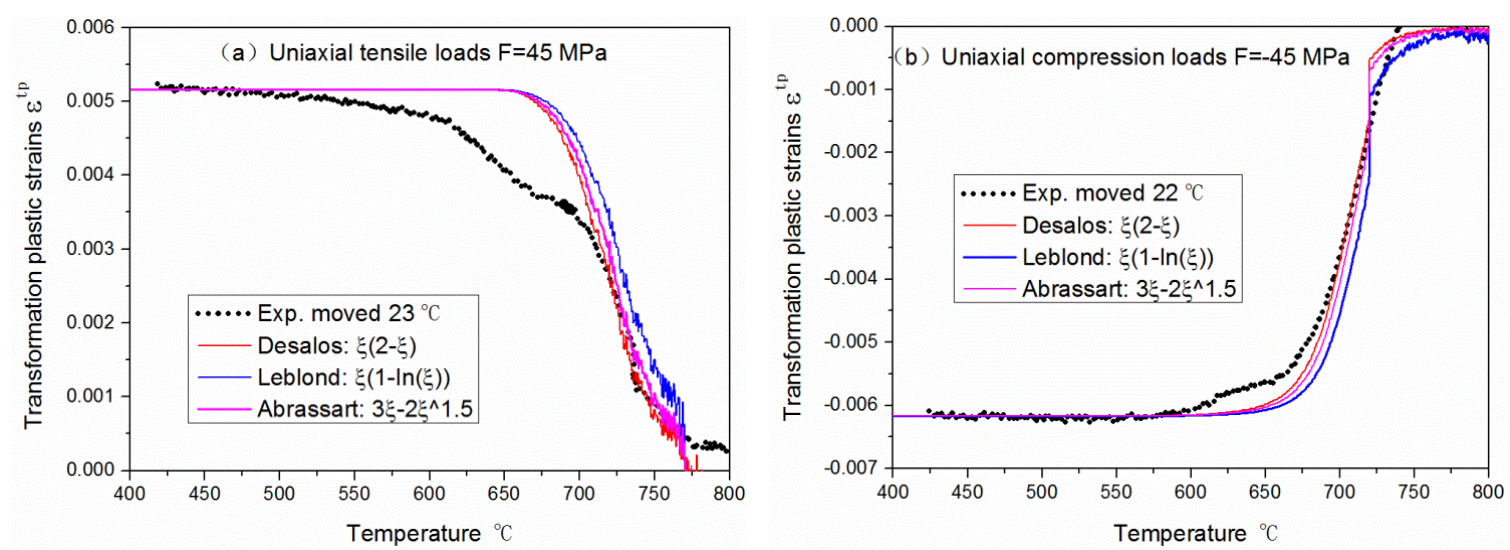

Figure 10. Comparison of the calculated and the corrected experimental values for ferrite transformation in H420LA steel.

\section{Conclusions}

In this paper, the kinetics and transformation plasticity for H420LA steel were considered. The following conclusions can be drawn:

(1) The ferrite transformation is inhibited by uniaxial loads (less than the yield strength of the parent phase) at the initial stage of the transformation during the continuous cooling process. This may be result of the elastic strain energy increase due to the external stress. Therefore, the anisothermal transformation curves are displaced to lower temperatures. Whereas, as the applied load increases, the activation energy, $Q$, which governs the transformation from the mother phase to the new phase, decreases, and the resulting the phase transformation hysteresis time is shortened. For H420LA ferrite transformation, the hysteresis time is $7.5 \mathrm{~s}$ under the $15 \mathrm{MPa}$ uniaxial tensile stress, and it is shortened to $2.3 \mathrm{~s}$ under the $45 \mathrm{MPa}$ uniaxial tensile stress.

(2) The hysteresis effects change the starting and ending temperatures of the transformation, modify the kinetics, and also lead to a deviation of the transformation plasticity at the beginning of the transformation.

(3) A general kinetics formulation for ferrite transformation dependent on external stress during the continuous cooling process was developed. The transformation hysteresis effects caused by a uniaxial applied load were considered in this proposed model. The model exhibits good agreement with the experimental results.

(4) The transformation plasticity kinetics used in practical applications do not account for the transformation hysteresis effects caused by uniaxial applied load, which leads to an overestimation in the description of the transformation plasticity at the initial stage of the transformation. Considering the transformation hysteresis effects, the calculated value at $-45 \mathrm{MPa}$ was in good agreement with the experimental results, and the results at $45 \mathrm{MPa}$ approached the experimental value.

Author Contributions: W.D., Y.L., J.X., L.S. and T.L. conceived and designed the experiments; W.D. analyzed the data and wrote the paper.

Acknowledgments: The authors gratefully acknowledge the financial support from the Major Projects of Technological Innovation of Hubei Province (No. 2018AAA005) and HBIS Group Technology Research Institute.

Conflicts of Interest: The authors declare no conflict of interest.

\section{References}

1. Denis, S.; Gautier, E.; Simon, A.; Beck, G. Stress-Phase-Transformation Interactions-Basic Principles, Modeling, and Calculation of Internal Stresses. Mater. Sci. Technol. 1985, 1, 805-814. [CrossRef]

2. Fischer, F.D.; Reisner, G.; Werner, E.; Tanaka, K.; Cailletaud, G.; Antretter, T. A new view on transformation induced plasticity (TRIP). Int. J. Plast. 2000, 16, 723-748. [CrossRef] 
3. Johnson, W.A.; Mehl, R.F. Reaction Kinetics in Processes of Nucleation and Growth. Metall. Mater. Trans. A $1939,35,1-27$.

4. Avrami, M. Kinetics of Phase Change. I General Theory. J. Chem. Phys. 1939, 7, 1103-1112. [CrossRef]

5. Avrami, M. Kinetics of Phase Change. II Transformation-Time Relations for Random Distribution of Nuclei. J. Chem. Phys. 1940, 8, 212-224. [CrossRef]

6. Fernandes, F.M.B.; Denis, S.; Simon, A. Mathematical model coupling phase transformation and temperature evolution during quenching of steel. Mater. Sci. Technol. 1985, 1, 838-841. [CrossRef]

7. Magee, C.L. Transformation Kineties, Micro-Plasticity and Aging of Martensite in Fe-3Ni. Ph.D. Thesis, Carnegie Institute of Technology, Pittsburgh, PA, USA, 1966.

8. Fischer, F.D. A micromechanical model for transformation plasticity in steels. Acta Metall. Mater. 1990, 38, 1535-1546. [CrossRef]

9. Greenwood, G.W.; Johnson, R.H. The Deformation of Metals under Small Stresses during Phase Transformations. Proc. R. Soc. Lond. Ser. A Math. Phys.Sci. 1965, 283, 403-422.

10. Taleb, L.; Petit, S. New investigations on transformation induced plasticity and its interaction with classical plasticity. Int. J. Plast. 2006, 22, 110-130. [CrossRef]

11. Taleb, L.; Cavallo, N.; Waeckel, F. Experimental analysis of transformation plasticity. Int. J. Plast. 2001, 17, 1-20. [CrossRef]

12. Leblond, J.B.; Devaux, J.; Waeckel, F. Mathematical modelling of transformation plasticity in steels I: Case of ideal-plastic phases. Int. J. Plast. 1989, 5, 551-572. [CrossRef]

13. Jiang, W.C.; Woo, W.; Wan, Y.; Lou, Y.; Xie, X.F.; Tu, S.T. Evaluation of through-thickness residual stresses by neutron diffraction and finite-element method in thick weld plates. J. Press. Vess. Technol. 2017, 139, 031401. [CrossRef]

14. Domański, T.; Bokota, A. The numerical model prediction of phase components and stresses distributions in hardened tool steel for cold work. Int. J. Mech. Sci. 2015, 96, 47-57. [CrossRef]

15. Liu, Y.; Qin, S.; Zhang, J.; Wang, Y.; Rong, Y.; Zuo, X.; Chen, N. Influence of Transformation Plasticity on the Distribution of Internal Stress in Three Water-Quenched Cylinders. Metall. Mater. Trans. A 2017, 48, 4943-4956. [CrossRef]

16. Hamelin, C.J.; Muránsky, O.; Smith, M.C.; Holden, T.M.; Luzin, V.; Bendeich, P.J.; Edwards, L. Validation of a numerical model used to predict phase distribution and residual stress in ferritic steel weldments. Acta Mater. 2014, 75, 1-19. [CrossRef]

17. Jiang, W.; Chen, W.; Woo, W. Effects of low-temperature transformation and transformation-induced plasticity on weld residual stresses: Numerical study and neutron diffraction measurement. Mater. Des. 2018, 147, 65-79. [CrossRef]

18. Weisz-Patrault, D. Multiphase model for transformation induced plasticity. Extended Leblond's model. J. Mech. Phys. Solids 2017, 106, 152-175. [CrossRef]

19. Ni, J.; Wang, X.; Gong, J.; Wahab, M.A. A multi-phase model for transformation plasticity using thermodynamics-based metallurgical algorithm. Int. J. Mech. Sci. 2018, 148, 135-148. [CrossRef]

20. Hu, G.; Cai, X. Fundamentals of Materials Science; Shanghai Jiaotong University Press: Shanghai, China, 2006; pp. 228-229.

21. Ferry, M.; Humphrey, J. Discontinuous subgrain growth in deformed and annealed $\{110\}<001>$ aluminium single crystals. Acta Mater. 1996, 44, 1293-1308. [CrossRef]

22. Gottstein, G.; Molodov, D.A.; Shvindlerman, L.S.; Winning, M. Grain Boundary Dynamics: A Novel Tool for Microstructure Control. Interface Sci. 2001, 9, 297-306. [CrossRef]

23. Jahanian, S.; Mosleh, M. The mathematical modeling of phase transformation of steel during quenching. J. Mater. Eng. Perform. 1999, 8, 75-82. [CrossRef]

24. Xu, Z. Martensitic Transformation and Martensite, 2nd ed.; Science Press: Beijing, China, 1999; pp. 593, 700.

25. Xu, Z. Effect of stress on bainitic transformation in steel. Acta Metall. Sin. 2004, 2, 113-119.

26. Yong, Q. Second Phases in Structural Steels; Metallurgical Industry Press: Beijing, China, 2006; pp. 258-293.

(C) 2019 by the authors. Licensee MDPI, Basel, Switzerland. This article is an open access article distributed under the terms and conditions of the Creative Commons Attribution (CC BY) license (http:/ / creativecommons.org/licenses/by/4.0/). 